\title{
Capacity of the Multiple Access Channel in Energy Harvesting Wireless Networks
}

\author{
R.A. Raghuvir, Dinesh Rajan and M.D. Srinath \\ Department of Electrical Engineering \\ Southern Methodist University \\ Dallas, TX 75275-0338 \\ Email: \{rraghuvi,rajand,mds\}@lyle.smu.edu
}

\begin{abstract}
In this paper, we obtain the capacity region for the AWGN Multiple Access Channel (MAC) with transmitters equipped with energy harvesters. We next obtain the sum-of-rates capacity for the flat fading Multiple Access Channel with energy harvesting transmitters. We then develop low delay, adaptive transmission strategies that surprisingly achieve near optimal performance under finite battery resource constrained settings. We study the effect of asymmetries in the energy harvesting and characterize the achievable performance.
\end{abstract}

\section{INTRODUCTION}

The ergodic capacity of the additive white Gaussian noise (AWGN) channel with an average transmit power constraint, was first given by Shannon in his pioneering work [1]. Subsequently, the capacity of several multiuser fading channels have been widely investigated (see [2] for an excellent review). Recently, several information-theoretic analysis of channels with an energy harvesting mechanism at the transmitter have appeared in the literature. The capacity of a point-to-point AWGN channel with an energy harvester at the transmitter is given in [3], [4], [5]. In contrast to traditional capacity results which are typically derived under an average transmit power constraint, in the energy harvesting scenario, the energy harvesting process induces a constraint on the transmit power at each time epoch. In these works, the capacity is shown to be upper bounded by the capacity of a point-to-point system without an energy harvester at the transmitter, but with average power constraint equal to the recharge rate. In this paper we investigate the performance of a multiple access channel (MAC) when the individual transmitters are equipped with energy harvesters and a finte energy storage element such as a battery.

The main contributions of this paper are as follows. First, we consider the simple AWGN-MAC with an infinite capacity battery and characterize its capacity region. Interestingly, we show that this capacity region is the same as the capacity region of a MAC with equivalent average power constraints for each user. Second, we consider the flat fading MAC and characterize the maximum sum rate that is achieveable with independent energy harvesting processes and an infinite capacity battery at each user. It is shown that this maximum sum rate equals the maximum sum rate with equivalent sum power constraint. Third, we study the effect of finite size batteries at each of the transmit nodes. In such cases the achievable rates depends on: i) battery sizes, ii) energy harvesting process, and iii) channel fading process. We study several low complexity adaptive transmission strategies and show that they achieve near optimal performance under resource constrained settings. Finally, we also study the effect of assymetries in the energy harvesting and channel fading processes for the various users on the achievable performance.

\section{A. Related Work}

In a flat fading channel, the channel power gain or channel side information (CSI), stays constant during a symbol interval. The capacity of such a channel with CSI known to both transmitter and receiver, and with average power constraint on the transmitter, is given in [6]. The power allocation that achieved capacity was shown to be water-filling in time, which takes advantage of good channel conditions. More power is allocated for higher channel gains and less power for lower channel gains. To find the capacity of a fading channel in energy harvesting systems, the work in [5] was extended in [7]. The optimal power allocation in this case is also shown to be water-filling in time, with the average power constraint equal to the average recharge rate.

In a multi-user flat fading multiple access channel, the optimal power control that maximizes the sum-of-rates is shown to be a TDMA-like approach [8] popularly known as opportunistic scheduling [9]. In this approach, in the symmetric case where the average power constraint is the same for all users, only the user that enjoys the best channel power gain is allowed to transmit and the assigned power is computed by temporal water-filling, similar to the single user scenario [6]. In [10], opportunistic scheduling is considered in energy harvesting networks with emphasis on queue stability rather than sum-of-rates capacity. The variation of mean delay with the packet arrival rate is characterized for a few decentralized algorithms.

The results in this paper are applicable in a wide range of scenarios, for instance a wireless sensor network, where each node has an energy harvester and is communicating with a fusion center. We consider centralized scheduling and power control at the fusion center, in which the fusion center instructs each sensor node as to when to transmit and the transmit power level to be used. We also assume that a feedback channel 
provides accurate information of the instantaneous channels and battery conditions of the various users to the fusion center.

The rest of the paper is organized as follows. Section II gives the capacity region for the AWGN-MAC. Section III provides the optimal strategy that maximizes sum-of-rates capacity for a flat fading MAC. In Section IV we describe three low delay, adaptive transmission strategies. In Section V we study the effect of finite size batteries on throughput and characterize the performance of the low delay, adaptive transmission strategies. Section VI concludes the paper.

\section{CAPACITY OF THE AWGN MAC}

Consider a discrete time multiple access channel with $K$ users transmitting to a single receiver. Let $X_{i, j}$ represent the signal transmitted by user $i$ at time instant $j$. The received signal $Y_{j}$ at the receiver is given by

$$
Y_{j}=\sum_{i=1}^{K} \alpha_{i, j} X_{i, j}+N_{j}
$$

where $\alpha_{i, j}$ is the channel gain of user $i$ at time $j$ and $N_{j}$ is the additive noise at the receiver with variance $\sigma^{2}$. For a nonfaded MAC, $\alpha_{i, j}=\alpha_{i} \forall j$. Further, if the channels of all the users are symmetric then $\alpha_{i}=\alpha_{k}, \forall i, k \in\{1,2, \ldots K\}$.

Each user $i$ has an energy harvesting mechanism and the energy harvested at time $j$ is denoted by $E_{i, j}$ Joules. The energy harvesting process for each user is assumed to be ergodic and independent of the energy harvesting of the other users. Let the symbol period equal $T$. Assume that each user also has a battery with capacity equal to $B$ Joules in which the harvested energy is stored. In the initial capacity results, we set $B \rightarrow \infty$. In the subsequent section, we quantify the effect of finite battery sizes. This energy harvesting process induces a natural constraint on the transmit power,

$$
\sum_{j=1}^{m} X_{i, j}^{2} T \leq \sum_{j=1}^{m} E_{i, j} \quad \forall m, i=1,2, \ldots K
$$

For notational convenience, we define $C(x)=0.5 \log (1+x)$.

We first consider the special case of a AWGN-MAC and characterize the capacity region in the following theorem.

Theorem 1: (Capacity Region of AWGN channel with Average Power Constraint) Consider the $K$-user AWGN MAC with independent random energy arrivals for each of the $K$ users represented by the vector $\left[E_{1} E_{2} \ldots E_{K}\right]$. Let $P_{i}=\mathbf{E}\left[E_{i}\right] / T$ represent the average power constraint for user $i$. Then, the transmission rates $R_{i}$ for user $i$ are given by the closure of the convex hull of the rate vectors satisfying

$$
\sum_{i \in S} R_{i} \leq C\left(\frac{\sum_{i \in S} \alpha_{i}^{2} P_{i}}{\sigma^{2}+\sum_{i \in S^{c}} \alpha_{i}^{2} P_{i}}\right) \forall S \subseteq\{1,2, \cdots, K\}
$$

Proof:

Upper bound: It is clear that the rates in (3) is the upper bound of a Gaussian MAC without energy harvesting and an average power constraint $P_{i}, \forall i$. Hence, (3) is also an upper bound on the achieveable rates with energy harvesting nodes. Achievability:
For each user $i$ with energy harvesting, the maximum achieveable rate is given in [3], [4], [5] as

$$
R_{i}=C\left(\frac{\alpha_{i}^{2} P_{i}}{\sigma^{2}}\right)
$$

This rate is achieved using the save-and-transmit scheme employed in [3], [4]. Rather than ensuring the cumulative power constraint in (2) is satisfied for every time instant, the random nature of the energy arrivals is smoothed out by storing the energy in an infinite size energy buffer. The transmitter essentially waits for $s(n)$ time slots before the start of transmission. It is shown in [3], [4] that $s(n) \in o(n)$ i.e. $s(n)$ belongs to a class of functions $o(n)$ that scale slower than $n$. This guarantees adequate energy is available in the energy buffer of each user and avoids the case of the encoder not being able to transmit a codeword due to insufficient energy. Further, in the limit of large $n$ the loss in rate due to nontransmission during the first $o(n)$ symbols reduces to 0 . We now extend this idea to the multi-user scenario.

Consider any set $S \subseteq\{1,2, \cdots, K\}$ of users. Depending on the arrival process for each user $i \in S$, using the results from [] there exists a time denoted by $s_{i}$ such that if user $i$ is silent for the first $s_{i}$ time instants, it can subsequetly transmit at average power $P_{i}$ for the remaining $n-s_{i}$ time instants. Now, consider $\tilde{s}(S)=\max \left(s_{i}, i \in S\right)$. Since each $s_{i} \in o(n)$, this implies that $\tilde{s}(S) \in o(n)$. Clearly, if all users $i \in S$ are silent for the first $\tilde{s}(S)$ time instants, they can subsequently transmit at their average powers. Now, let each user $i \in S$ transmit using an independent Gaussian codebook (the traditional MAC capacity achieving codebook without energy harvesting) of size $\left(2^{(n-\tilde{s}) R_{i}}, n-\tilde{s}\right)$. At the receiver, using the successive decoding method any of the corner points of the capacity region can be achieved by carefully designating the order of decoding. Thus, any set of rates satisfying (3) can be achieved with arbitrarily small probability of error. Due to the transmitters remaining silent for duration $\tilde{s}$, the average rate achieved is lower and equals $\frac{n-\tilde{s}}{n} R_{i} \rightarrow R_{i}$ in the limit of large $n$.

\section{CAPACITY OF THE FLAT FADING MAC}

The next channel we consider is the frequency flat fading MAC where the fading process is independent for the various users. We let $\alpha_{i}$ have a Rayleigh distribution and consquently the signal-to-noise (SNR) ratio $\gamma_{i}=\alpha_{i}^{2} P_{i} / \sigma^{2}$ is exponentially distributed with PDF, $p\left(\gamma_{i}\right)$, given by

$$
p\left(\gamma_{i}\right)= \begin{cases}\frac{1}{\gamma_{s i}} \exp \left(\frac{\gamma_{i}}{\gamma_{s i}}\right), & \gamma_{i}>0 \\ 0, & \text { otherwise }\end{cases}
$$

where $\gamma_{s i}=\frac{\mathbf{E}\left[E_{i}\right] \mathbf{E}\left[\alpha_{i}^{2}\right]}{T \sigma^{2}}$ represents the average receive SNR of the $i$ th user.

Theorem 2: (Sum-of-Rates Capacity of flat fading channel with average power constraint) Consider the $K$ user fading MAC with independent random energy arrivals for each of the $K$ users represented by the vector $\left[E_{1} E_{2} \ldots E_{K}\right]$. Let $P_{i}=$ 
$\mathbf{E}\left[E_{i}\right] / T$ represent the average power constraint for user $i$. The maximal sum rate is given by

$$
C_{p c}=\frac{1}{2} \iint \cdots \int \log _{2}\left[1+\sum_{i=1}^{K} \mu_{i}(\underline{\gamma}) \gamma_{i}\right] p(\gamma) d \underline{\gamma},
$$

where, the optimal power control policy is given by (7a).

Proof:

For the non-energy harvesting scenario where the users have infinite energy at their disposal but subject to average power constraints, in a landmark paper, it was shown in [8] that the optimal power control law that maximizes the sum-of-rates capacity was opportunistic scheduling. Only one user accesses the channel over the entire bandwidth at any gliven time. This user is the one with the best channel gain, provided that channel gain is above a certain cut-off threshold. This power control law, taking all the users into account was shown to be

$$
\mu\left(\underline{\gamma_{i}}\right)= \begin{cases}\frac{1}{\lambda_{i}}-\frac{1}{\gamma_{i}}, & \gamma_{i}>\lambda_{i}, \gamma_{i}>\frac{\lambda_{i}}{\lambda_{j}} \gamma_{j}, j \neq i(7 \mathrm{a}) \\ 0, & \text { otherwise. }\end{cases}
$$

where $\lambda_{i}$ is the cut-off threshold for user $i$ and $\gamma=$ $\left[\gamma_{1} \gamma_{2} \cdots \gamma_{K}\right]$ is the instantaneous received power of all the users.

For the energy harvesting scenario, we again employ the save-and-transmit scheme of [4]. We let all the users wait for $\tilde{s}$ time slots before transmission. This save and transmit policy guarantees adequate energy is available in the energy buffer of each user and avoids the case of the encoder not being able to transmit a codeword due to insufficient energy. Then the results from [8] are directly applicable and the same power control law using $7 \mathrm{a}$ and $7 \mathrm{~b}$ can maximize the sum-of-rates capacity. As in the Gaussian case, the marginal loss in rate $\left(\frac{\tilde{s}}{n} \rightarrow 0\right.$, as $n \rightarrow \infty$ ) due to the nodes being silent during the initial portion of the scheme goes to zero.

In the symmetric case where all the users have the same recharge rate and consequently the same average power constraint, the cut-off threshold can be calculated from equation (14) of [8] as:

$$
\sum_{i=1}^{K}(-1)^{i-1}\left(\begin{array}{c}
K \\
i
\end{array}\right)\left[\frac{e^{-i \lambda / \gamma_{s}}}{\lambda}-\frac{i}{\gamma_{s}} E i\left(\frac{i \lambda}{\gamma_{s}}\right)\right]=K,
$$

where $E i($.$) is the first order exponential integral given by$

$$
E i(x)=\int_{x}^{\infty} \frac{1}{e^{t} t} d t
$$

The corresponding sum-of-rates capacity is given by

$$
C=\frac{1}{2 \ln 2} \sum_{i=1}^{K}(-1)^{i-1}\left(\begin{array}{c}
K \\
i
\end{array}\right) \operatorname{Ei}\left(\frac{i \lambda}{\gamma_{s}}\right) .
$$

For the asymmetric case where the users have different recharge rates, the channel cut-off threshold can be calculated usig equation (12) of [8] as:

$$
\frac{1}{\gamma_{s i}} \int_{\gamma_{i}}^{\infty} e^{-\gamma_{i} / \gamma_{s i}} \prod_{j=1, j \neq k}^{K}\left(1-e^{-\left(\frac{\lambda_{j}}{\lambda_{i}}\right) \frac{\gamma_{i}}{\gamma_{s j}}}\right) d \gamma_{i}=1 .
$$

\section{LOW-DELAY ADAPTIVE TRANSMISSION STRATEGIES}

In Section II and III we obtained the capacity of the AWGN MAC and the frequency flat MAC under the assumption of infinite battery size. The optimal capacity achieving strategy uses a store and transmit policy which is asymptotically optimal. However, it is not clear what the performance of those schemes are with finite battery sizes and low or no delay in transmissions. In this Section, we describe three low-delay, adaptive transmission strategies for the flat fading MAC. In Section V we characterize the throughput achievable when finite size limitations are imposed on the energy buffers.

\section{A. Single-user channel adaptive transmission - (SUCA)}

In this adaptive transmission strategy, the receiver selects the user with the best channel according to the optimal power control as described in Theorem 2. However, the transmitters do not wait to allow their energy buffers to accumulate energy prior to commencement of transmission, since with a finite battery size some users may end up wasting energy. Consequently, the user $i$ with the best channel may not have enough energy in their battery to transmit at the desired power level. In that case the transmission is done with any residual battery energy remaining in that user's energy buffer. The battery update is given by

$$
B_{i}(n+1)=B_{i}(n)+E_{i}(n)-\mu_{i}(n) T
$$

Formally,

$$
\mu_{i}\left(\underline{\gamma_{i}}\right)=\min \left\{\frac{B_{i}}{T},\left(\frac{1}{\lambda_{i}}-\frac{1}{\gamma_{i}}\right)\right\}
$$

\section{B. Group-power channel adaptive transmission - (GPCA)}

In this adaptive transmission strategy, the receiver first selects the user with the best channel and uses the optimal power control as described in Theorem 2. However, if the selected user does not have sufficient energy in its battery to transmit according to the optimal power control, the user with the next best channel is also selected and power is allocated from its battery. The process continues either till the sum power from the subset of selected users equals the power required per the optimal power control or till all users have been selected. Transmission is then done by the group of users selected.

Denoting the selected group of users as $S_{c}$, formally,

$$
\mu_{i}\left(\underline{\gamma_{i}}\right)=\min \left\{\frac{\sum_{i \in S_{c}} B_{i}}{T},\left(\frac{1}{\lambda_{i}}-\frac{1}{\gamma_{i}}\right)\right\}
$$

\section{Group-power battery adaptive transmission - (GPBA)}

In this adaptive transmission strategy, the receiver first selects the user with the highest battery energy. An attempt is made to allocate power from this user's battery to satisfy the sum power constraint, without consideration for the optimal power control. However, if the channel gain of the selected user is lower than the cut-off threshold specified in Theorem 2 , the user with the next highest battery energy is selcted. The process is repeated either till the sum power from the selcted 
users equals the sum power constraint or till all the users have been selected. Transmission is then done by the group of users selected.

Denoting the selected group of users as $S_{B}$, formally,

$$
\mu_{i}\left(\underline{\gamma_{i}}\right)=\min \left\{\frac{\sum_{i \in S_{B}} B_{i}}{T}, \sum_{i=1}^{K} P_{i}\right\}
$$

\section{PERformance With FINITE BATteries}

In this Section we evaluate the sum-of-rates achieved by the three low-delay adaptive transmission strategies outlined in Section IV for the frequency flat Rayleigh channel and compare their performance under finite battery constraints.

An exponential distribution is selected for the energy harvesting process. Symmetric conditions are assumed for the users whereby each user has the same energy harvesting statistics and the same battery capacity. We also set $T=1$. Further, we assume each user undergoes same channel variations on average. The channel cut-off for each user group $\mathrm{K}=2,4$ and 16 and the upper bound on the sum-of-rates capacity is computed by numerically evaluating (8) and (10) respectively.

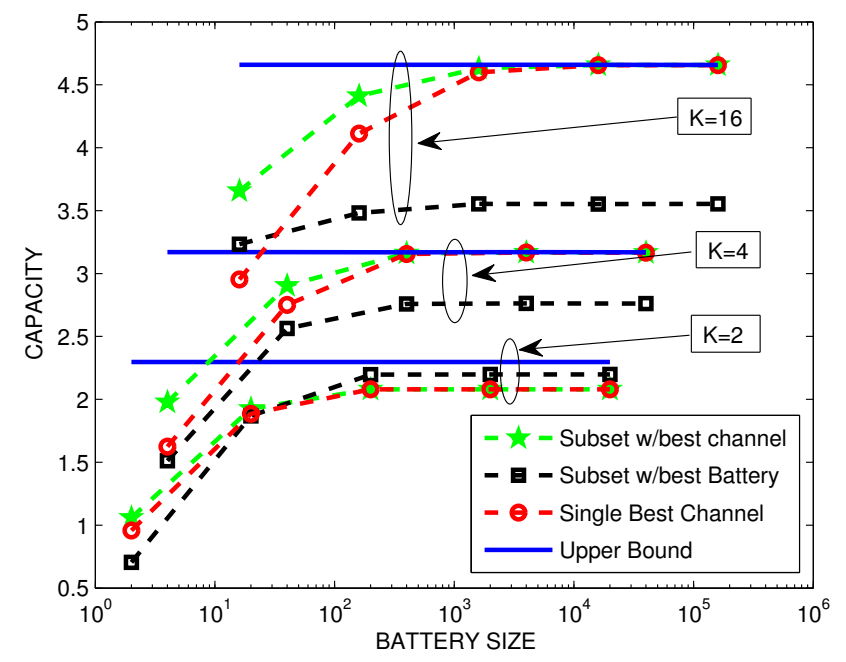

Fig. 1. $C$ versus $B$ for $K=2,4,16$. Performance of various low delay adaptive transmission schemes are shown. The solid lines represent the respective upper bound on the sum-of-rates capacity. The recharge rates of the users are identical and equal to the average power constraint $P=10$ watts.

Figure 1 shows the variation of average sum-of-rates capacity with battery size for user groups of 2, 4 and 16. Each user has the same recharge rate of 10 Joules. Hence the average power constraint on each user is 10 Watts. Remarkably, the channel adaptive schemes SUCA and GPCA acheive the sumrate for $\mathrm{K}=4$ and 16 even for reasonably small battery sizes. For the user group $\mathrm{K}=2$, none of the strategies achieve the sumrate which leads us to conjencture that the save-and-transmit scheme is required to achieve the sum-rate. The interesting result however is that the battery adaptive scheme GPBA achieves higher sum-rate in this case. From our simulations we find that a general rule-of-thumb for selecting the battery size is about $10 E_{\text {sum }}$ Joules where $E_{\text {sum }}$ is the energy required to deliver $P_{\text {sum }}$ Watts and $P_{\text {sum }}$ is the sum-of-average power contraint.

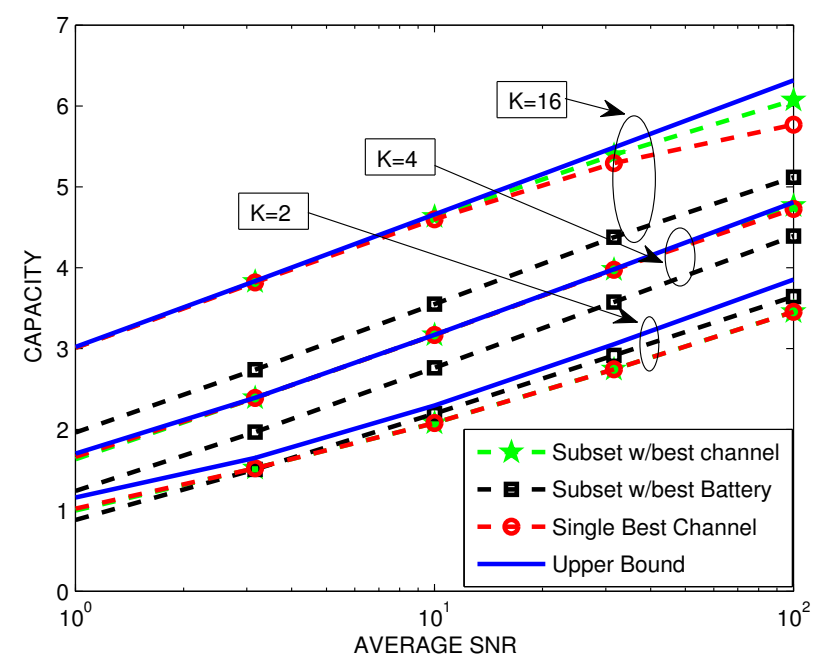

Fig. 2. $C$ versus $S N R$ for $K=2,4,16$. Performance of various low delay adaptive transmission schemes are shown. The solid lines represent the respective upper bound on the sum-of-rates capacity. The battery size is fixed at $1600 \mathrm{~J}$.

Figure 2 shows the variation of sum-of-rates capacity with average SNR. The SNR is proportional to the average recharge rate. The battery size is fixed at $1600 \mathrm{~J}$ which is selected to equal the sum-power at the highest $\mathrm{SNR}=100$ for $\mathrm{K}=16$ users. This size is clearly small for the 16 user case and as a result, none of the strategies achieve sum-of-rates capacity for higher SNR values, as can be seen from the Figure. The insufficient battery size however brings out an interesting observation with regards to the Group-Power Channel Adaptive (GPCA) strategy. For the 16 user case at high SNR this strategy outperforms Single-User Channel Adaptive (SUCA) strategy. This result is because the group strategy salvages more energy from a subset of users in each channel use thereby reducing the energy lost due to the finite battery size. As in Figure 1, the battery adaptive GBPA strategy achieves poor performance for $\mathrm{K}=4$ and 16 user case but better performance for the $\mathrm{K}=2$ user case than GPCA and SUCA.

Figure 3 shows the variation of sum-of-rates capacity with average SNR for the two user case with asymmetric energy harvesters. The Figure shows increasing energy harvesting capability for user 2 (and consequently an increasing power constraint), while user 1 has a fixed energy harvester. We compare the performance of SUCA and GPBA schemes against the achievable upper bound. We fix the battery size to be large to observe the achievable performance with low delay. The channel thresholds are computed by solving two non-linear equations simultaneously (equation (12) in [8]). Since resulting power control law calls for picking the best user per $7 \mathrm{a}$ and $7 \mathrm{~b}$, the GPBA reduces to a single user transmission strategy. As can be seen from the Figure, performance of SUCA is 


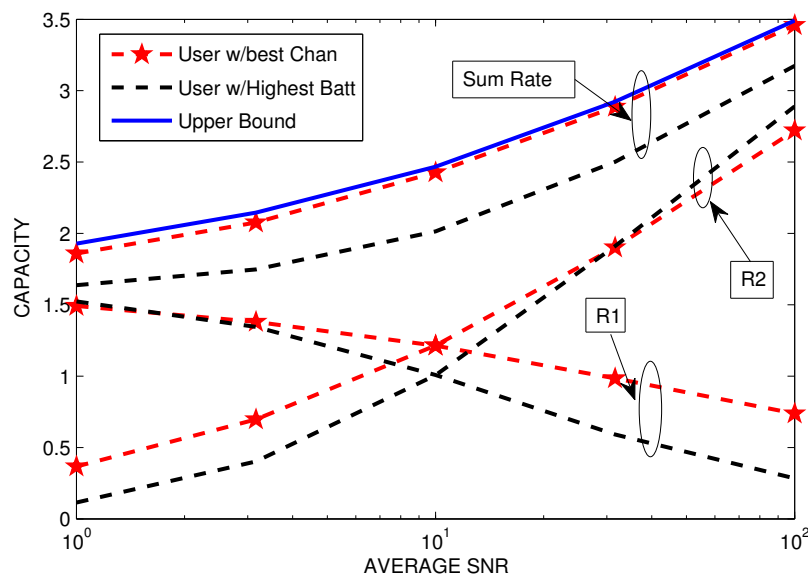

Fig. 3. $C$ versus $S N R$ for $\mathrm{K}=2$ with asymmetric energy harvesters. $E_{1}=10 \mathrm{~J}$ and as a result $P_{1}$ is fixed with varying $E_{2}$ (and consequently varying $P_{2}$ ) Performance of various low delay adaptive transmission schemes are shown The solid lines represent the respective upper bound on each user's capacity as well as the sum-of-rates capacity. The battery size is fixed at a large value.

better in the asymmetric case than in the symmetric case. In the latter case, even for large battery size $\left(10^{4} \mathrm{~J}\right)$, the sum-ofrates capacity was not approached (see Figure 1). While the SUCA strategy approaches the sum-of-rates capacity in the asymmetric case, performance of the GPBA strategy is worse than the SUCA performance in the large battery regime. The opposite was observed in the symmetric case (again see Figure $1)$.

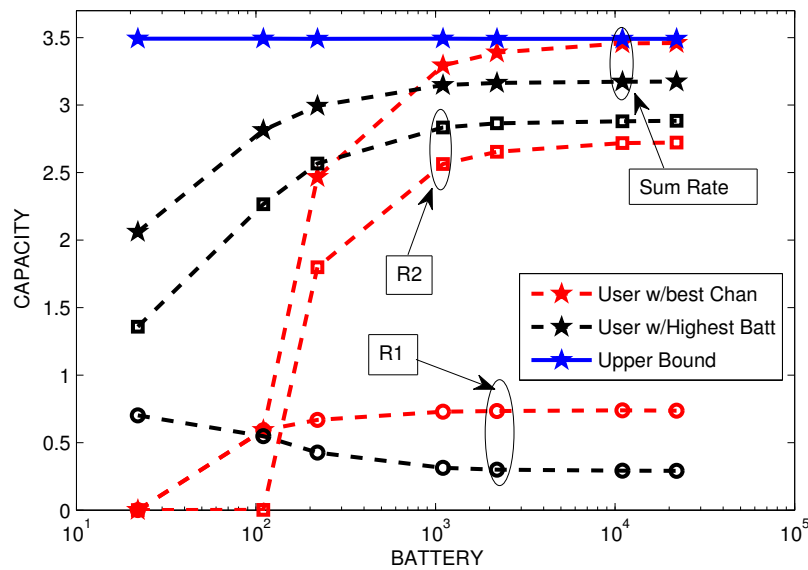

Fig. 4. $C$ versus $B$ for $\mathrm{K}=2$ with asymmetric energy harvesters. $E_{1}=10 \mathrm{~J}$ and $E_{2}=100 \mathrm{~J}$. Performance of various low delay adaptive transmission schemes are shown. The solid lines represent the respective upper bound on each user's capacity as well as the sum-of-rates capacity.

Figure 4 shows variation of sum-of-rate capacity with battery size for two users with asymmetric harvesters for the two low delay adaptive transmission strategies namely SUCA and GPBA. User 1 and User 2 have harvesters capable of generating $10 \mathrm{~J}$ and $100 \mathrm{~J}$ on average. As a result the power constraints for user 1 and 2 are respectively $10 \mathrm{~W}$ and 100
W. The battery size is varied upto $10^{5} \mathrm{~J}$. Just as in the symmetric case (see Figure 2), the GPBA scheme achieves better performance for smaller battery sizes. This is because the power required per the optimal power control law is not available due to the small battery sizes of the individual users. As the battery size increases, SUCA strategy performs better due to the availablity of energy to implement the optimal power control law and take advantage of the favorable channel opportunistically.

\section{CONCLUSIONS}

In this paper we obtained the entire AWGN-MAC capacity region with energy harvesting transmitters. Next for the flat fading channel, we obtained the sum-of-rates capacity with energy harvesting transmitters. Then for the multi-user symmetric case, where each user has identical energy harvesting capability, we studied three low delay, adaptive transmission schemes that acheived near optimal performance under resource constrained settings. Finally for the two-user asymmetric case, where each user has different energy harvesting capabilities, we characterized performance of two low delay adaptive transmission schemes both with large batteries and smaller size batteries. Future work would involve developing adaptive transmission schemes that incorporate fairness towards the users.

\section{ACKNOWLEDGMENT}

The authors would like to thank Professor Sennur Ulukus for providing reference [4] which helped in formulating the proofs in this paper.

\section{REFERENCES}

[1] C. Shannon, "A mathematical theory of communication," The Bell System Technical Journal, vol. 27, no. 7, pp. 379-423, 1948.

[2] E. Biglieri, J. Proakis, and S. Shamai, "Fading channels: Informationtheoretic and communications aspects," Information Theory, IEEE Transactions on, vol. 44, no. 6, pp. 2619-2692, 1998.

[3] O. Ozel and S. Ulukus, "Information-theoretic analysis of an energy harvesting communication system," in 2010 IEEE 21st International Symposium on Personal, Indoor and Mobile Radio Communications Workshops (PIMRC Workshops). IEEE, 2010, pp. 330-335.

[4] —_, "Achieving awgn capacity under stochastic energy harvesting," Preprint, 2011.

[5] R. Rajesh, V. Sharma, and P. Viswanath, "Information capacity of energy harvesting sensor nodes," Arxiv preprint arXiv:1009.5158, 2010.

[6] A. Goldsmith and P. Varaiya, "Capacity of fading channels with channel side information," IEEE Transactions on Information Theory, vol. 43, no. 6, pp. 1986-1992, 1997.

[7] R. Rajesh and V. Sharma, "Capacity of fading gaussian channel with an energy harvesting sensor node," Arxiv preprint arXiv:1010.5416, 2010.

[8] R. Knopp and P. Humblet, "Information capacity and power control in single-cell multiuser communications," in IEEE International Conference on Communications, 1995. ICC'95 Seattle,'Gateway to Globalization', 1995, vol. 1. IEEE, 1995, pp. 331-335.

[9] A. Goldsmith, Wireless communications. Cambridge Univ Press, 2005.

[10] V. Sharma, U. Mukherji, and V. Joseph, "Efficient energy management policies for networks with energy harvesting sensor nodes," in 46th Annual Allerton Conference on Communication, Control, and Computing. IEEE, 2008, pp. 375-383. 